\title{
Analytical solution of a one-dimensional Ising model with zero temperature dynamics
}

\author{
A. Prados* and J. Javier Brey ${ }^{\dagger}$ \\ Física Teórica, Facultad de Física, Universidad de Sevilla, Apdo. de Correos 1065, E-41080 Sevilla, Spain
}

(November 2, 2018)

\begin{abstract}
The one-dimensional Ising model with nearest neighbour interactions and the zero-temperature dynamics recently considered by Lefevre and Dean -J. Phys. A: Math. Gen. 34, L213 (2001)- is investigated. By introducing a particle-hole description, in which the holes are associated to the domain walls of the Ising model, an analytical solution is obtained. The result for the asymptotic energy agrees with that found in the mean field approximation.

PACS: $05.50+q$, 05.70.Ln, 45.70.Cc
\end{abstract}

\section{INTRODUCTION}

Ising systems are often used as simple models in many different fields of statistical physics. In particular, the one-dimensional Ising model with nearest neighbour interactions and Glauber dynamics [i] is ubiquitous. It shows glass-like behaviour, including non-exponential relaxation [2 7], a laboratory glass transition in cooling processes [8,9], hysteresis effects in thermal cycling experiments [9], and aging effects at low temperatures 10 13]. When a weak oscillating external field is applied, the amplitude of the induced magnetization presents a maximum as a function of the temperature, a behaviour resembling stochastic resonance [14,15].

Other kind of important Ising systems are those with "facilitated" dynamics. They were introduced in a pioneering work by Fredrickson and Andersen [16.17, in the context of the relaxation of structural glasses. In these models, a given spin can only flip if its nearest neighbours are in a certain subset of all their possible configurations. The one-dimensional Ising model with facilitated dynamics (1SFM) has been extensively studied 18 24. Although, in general, it is not possible to find an analytical solution of these facilitated Ising models, the 1SFM has been exactly solved at zero temperature [21,23]. Very recently, the 1SFM has been applied to study the compaction of vibrated granular systems. A hole (particle) is associated to any spin in its excited (ground) state. Trying to mimic what is done in real experiments [25,26], the tapping process is simulated in the following way. First, the system freely relaxes to a metastable state $(n=0)$ following the dynamics at zero temperature, in which the processes decreasing the density of particles are forbidden and any configuration with all the holes being isolated is an absorbent state of the dynamics 27. Then, the system is tapped, allowing the density to decrease. Afterwards, a new free relaxation at zero temperature is done, reaching another metastable state $(n=1)$. By repeating this process, a chain $(n=0,1,2, \ldots)$ of metastable configurations is obtained, with the density of the system increasing as a function of $n$.

An analogous approach to the problem of granular compaction has been carried out by Lefevre and Dean [28, 29]. Their model is again the one-dimensional Ising system with nearest neighbour interactions. Nevertheless, the usual Glauber dynamics [1] is not a good choice for simulating tapping processes. This is because there are no metastable states, and at zero temperature the system always reaches the perfectly ordered ferromagnetic phase. Then, other zero temperature single spin flip dynamics was considered, in which only the elementary events lowering the energy are possible. In this way, all the states composed of domains of $l$ parallel spins with length $l \geq 2$ are metastable, i. e., they are absorbent states for this dynamics. In such states, there is no spin antiparallel to both of its nearest neighbours, which are the only spins being able to flip. With this falling dynamics, the tapping process is simulated in the same way as described above.

In ref. [28], the authors claimed that the new zero temperature dynamics does not seem amenable to analytic solution. In fact, the usual way of solving the Glauber model, by constructing the hierarchy of equations for the two-spin moments, does not work. The aim of this paper is to show that it is possible to solve analytically the time evolution of the model by going to an equivalent particle-hole description of the Ising system. The holes are associated to the domain walls separating arrays of parallel spins (particles). Within this picture, the metastable states are those with all the holes being isolated, as in the one-dimensional facilitated Ising model.

\footnotetext{
*e-mail:prados@cica.es

${ }^{\dagger}$ e-mail:brey@cica.es
} 
The paper is organized as follows. In section II the Ising model is introduced, as well as the equivalent particle-hole description. Section III] is devoted to the analytical solution of the dynamics. A closed hierarchy of equations is derived for the probabilities $D_{r}$ of finding $r+1$ consecutive holes. The solution of this hierarchy is obtained by means of a generating function method. In particular, the asymptotic density of holes and energy in the metastable state are exactly calculated. The last section contains some final remarks.

\section{THE MODEL}

We consider the one-dimensional Ising model with nearest neighbour interactions and periodic boundary conditions. The hamiltonian of the system is

$$
\mathcal{H}=-J \sum_{i=1}^{N} \sigma_{i} \sigma_{i+1}
$$

where $J>0$ is the coupling constant, having dimensions of energy, $N$ is the number of spins, and $\sigma_{i}= \pm 1$. The time evolution of the system is described by a Markov process with single spin flip dynamics. Then, the probability $p(\boldsymbol{\sigma}, t)$ of finding the system in configuration $\boldsymbol{\sigma} \equiv\left\{\sigma_{i}\right\}$ at time $t$ obeys the master equation

$$
\frac{d}{d t} p(\boldsymbol{\sigma}, t)=\sum_{i}\left[W\left(\boldsymbol{\sigma} \mid R_{i} \boldsymbol{\sigma}\right) p\left(R_{i} \boldsymbol{\sigma}, t\right)-W\left(R_{i} \boldsymbol{\sigma} \mid \boldsymbol{\sigma}\right) p(\boldsymbol{\sigma}, t)\right]
$$

Here $R_{i} \boldsymbol{\sigma}$ is the configuration obtained from $\boldsymbol{\sigma}$ by flipping the $i$-th spin, and $W\left(R_{i} \boldsymbol{\sigma} \mid \boldsymbol{\sigma}\right)$ is the transition rate for that process. Following Lefevre and Dean [28], we consider a zero temperature dynamics in which only the spin flips lowering the energy are permitted. Namely, we take

$$
W\left(R_{i} \boldsymbol{\sigma} \mid \boldsymbol{\sigma}\right)=\frac{\alpha}{4}\left(1-\sigma_{i-1} \sigma_{i}\right)\left(1-\sigma_{i} \sigma_{i+1}\right)
$$

i. e., the transition rate equals $\alpha$ if the $i$-th spin is antiparallel to both of its nearest neighbours, and vanishes otherwise. While an exact solution of the usual Glauber dynamics can be found for all temperatures from the hierarchy of equations for the two-spin moments [1], the same procedure applied to this "falling" dynamics leads to a non-closed set of equations, since more complex moments, involving three and four spins, appear in them.

For the above reason, it is convenient to go to an equivalent particle-hole description of the Ising model. For each site $i$, we define a new variable

$$
m_{i}=\frac{1-\sigma_{i} \sigma_{i+1}}{2}
$$

When $m_{i}=1$ we will say that site $i$ is occupied by a hole, while if $m_{i}=0$ we will refer to site $i$ as being occupied by a particle. Thus, there is a hole at site $i$ if the spins $i$ and $i+1$ are antiparallel, while a particle corresponds to spins $i$ and $i+1$ being parallel. It follows that holes are associated to the domain walls separating arrays of parallel spins (particles). It is important to note that the number of holes is even for any configuration with periodic boundary conditions. In terms of the new variables, the hamiltonian of the system reads

$$
\mathcal{H}=J \sum_{i=1}^{N}\left(2 m_{i}-1\right)=-J N+2 J \sum_{i=1}^{N} m_{i}
$$

We define the average dimensionless energy per spin $\varepsilon$ as

$$
\varepsilon(t) \equiv \frac{\langle\mathcal{H}\rangle_{t}}{J N}=-1+\frac{2}{N} \sum_{i=1}^{N}\left\langle m_{i}\right\rangle_{t}
$$

where the angular brackets $\langle\ldots\rangle_{t}$ denote average with $p(\boldsymbol{\sigma}, t)$.

In the particle-hole description, the elementary events involve two adjacent sites. When the $i$-th spin flips, both $m_{i-1}$ and $m_{i}$ change their state. From Eq. (3), the transition rate $\omega\left(R_{i-1} R_{i} \boldsymbol{m} \mid \boldsymbol{m}\right)$ for this process is

$$
\omega\left(R_{i-1} R_{i} \boldsymbol{m} \mid \boldsymbol{m}\right)=\alpha m_{i-1} m_{i} .
$$


As $\alpha$ only determines the arbitrary time scale, we will set $\alpha=1$ in the following. The master equation for the probability $p(\boldsymbol{m}, t)$ of finding the system in configuration $\boldsymbol{m}$ at time $t$ reads

$$
\frac{d}{d t} p(\boldsymbol{m}, t)=\sum_{i}\left[\omega\left(\boldsymbol{m} \mid R_{i-1} R_{i} \boldsymbol{m}\right) p\left(R_{i-1} R_{i} \boldsymbol{m}, t\right)-\omega\left(R_{i-1} R_{i} \boldsymbol{m} \mid \boldsymbol{m}\right) p(\boldsymbol{m}, t)\right] .
$$

In the dynamics given by the transition rates in Eq. (7), only two nearest neighbour holes can turn into two particles. Therefore, it is clear that, after a long enough time period, the system will become stuck in a "metastable" state with all the holes being isolated, i. e., surrounded by two particles. Of course, the reached metastable state will depend on the initial configuration. This behaviour is reminiscent of the one showed by the $1 \mathrm{SFM}$ at $T=0$. In the latter, the system also reaches a metastable state with all the holes isolated, the specific final state depending on the initial condition 23.30]. Nevertheless, the model considered here and the 1SFM at zero temperature are not equivalent. The elementary dynamical events occurring in each of them are different. While a particle can be adsorbed on any empty site with at least one nearest neighbour hole in the 1SFM, in the present model two particles must be adsorbed simultaneously on two adjacent empty sites of the one-dimensional lattice.

\section{ANALYTICAL SOLUTION OF THE DYNAMICS}

In order to analyze the dynamics of the model we focus on the set of moments

$$
D_{r}(t) \equiv\left\langle m_{k} m_{k+1} \ldots m_{k+r}\right\rangle_{t}
$$

with $r \geq 1$. The local density of holes is given by the first term of this hierarchy,

$$
D_{0}(t)=\left\langle m_{k}\right\rangle_{t},
$$

and it is directly related with the energy of the system. From Eq. (6), it is

$$
\varepsilon=-1+2 D_{0} .
$$

Note that we are restricting ourselves to spatially homogeneous situations, so these moments $D_{r}$ do not depend on their first site $k$. From its own definition, the moment $D_{r}$ gives the probability of finding $r+1$ consecutive holes starting from a given arbitrary site of the lattice. Using the master equation (8) with the transition rates (7) one gets

$$
\frac{d}{d t} D_{r}(t)=-2 D_{r+1}(t)-r D_{r}(t)
$$

for all $r \geq 0$. This hierarchy can be solved by introducing the generating function

$$
G(x, t)=\sum_{r=0}^{\infty} \frac{x^{r}}{r !} D_{r}(t),
$$

from which all the moments $D_{r}(t)$ are easily obtained,

$$
D_{r}(t)=\lim _{x \rightarrow 0} \frac{\partial^{r}}{\partial x^{r}} G(x, t) .
$$

The hierarchy of equations (12) is equivalent to the following first order partial differential equation for the generating function:

$$
\partial_{t} G(x, t)=-(2+x) \partial_{x} G(x, t),
$$

which has to be solved with the initial condition

$$
G_{0}(x) \equiv G(x, 0)=\sum_{r=0}^{\infty} \frac{x^{r}}{r !} D_{r}(0) .
$$

By using standard techniques it is easily obtained 


$$
G(x, t)=G_{0}\left[(2+x) e^{-t}-2\right] .
$$

For large times the solution approaches the limit

$$
G(x, \infty)=G_{0}(-2)
$$

and taking into account Eq. (14),

$$
\lim _{t \rightarrow \infty} D_{0}(t)=G_{0}(-2), \quad \lim _{t \rightarrow \infty} D_{r}(t)=0, r \geq 1 .
$$

This shows that all the holes are isolated in the metastable state reached by the system in the long time limit. The probability of finding $r+1$ consecutive holes, which is equal to $D_{r}$, vanishes for $r \geq 1$. On the other hand, the asymptotic value of the density of holes $D_{0}(\infty)$ does depend on the initial state, being always smaller than its initial value. In this sense, it is worth noting that the hierarchy of equations (12) has as a stationary solution any constant value of $D_{0}$, and $D_{r}=0$ for $r \geq 1$.

Now, we are going to particularize the above results for a specific initial condition. Let us consider that the onedimensional Ising model is initially at equilibrium at a certain temperature $T$. Then, the probability that spins $i$ and $i+1$ are antiparallel is given by a function of the temperature $a(T)$,

$$
a(T)=\frac{\exp \left(-\frac{2 J}{k_{B} T}\right)}{1+\exp \left(-\frac{2 J}{k_{B} T}\right)},
$$

where $k_{B}$ is Boltzmann's constant. The value $a=1 / 2$ corresponds to $T \rightarrow \infty$, the system is at $t=0$ in a completely random configuration. From the form of the hamiltonian in the particle-hole description, Eq. (5), it follows that at equilibrium the $m_{k}$ variables are statistically independent, and

$$
D_{r}(0)=a^{r+1}
$$

By using Eq. (16), one gets the initial condition for the generating function,

$$
G_{0}(x)=a e^{a x},
$$

and Eq. (17) yields

$$
G(x, t)=a \exp \left\{a\left[(2+x) e^{-t}-2\right]\right\} .
$$

Now the expressions for the moments $D_{r}(t)$ can be directly calculated from Eq. (14),

$$
D_{r}(t)=a^{1+r} \exp \left[-r t+a\left(2 e^{-t}-2\right)\right] .
$$

As discussed in the previous paragraph, all the moments $D_{r}$ with $r \geq 1$ vanish in the long time limit. Moreover, the asymptotic density of holes is

$$
D_{0}(\infty)=a e^{-2 a}
$$

which depends on the initial temperature of the system. Equivalently, from Eq. (11) the specific dimensionless energy $\varepsilon(\infty)$ in the metastable state reads

$$
\varepsilon(\infty)=-1+2 a e^{-2 a}
$$

The mean field calculation presented by Lefevre and Dean 28,29 leads to the same expression (see also 31]). The asymptotic energy $\varepsilon(\infty)$ is maximal for $a=1 / 2$, i. e., when the system starts from the completely random configuration. This is in contrast with the behaviour found for the 1SFM. In the latter, if the initial condition is taken as the equilibrium state for a given value of the temperature $T$, the asymptotic density of holes is a monotonic function of the initial density of holes [23]. 


\section{FINAL REMARKS}

In this work we have studied the time evolution of the one-dimensional Ising model with the zero temperature dynamics recently considered by Lefevre and Dean [28,29]. In this dynamics only transitions decreasing the energy of the system are allowed. The difference with the usual zero temperature Glauber dynamics [1] is that flips of spins parallel to one of its neighbours and antiparallel to the other one are forbidden. In other words, the possibility of a diffusive motion of the domain walls is eliminated.

When trying to compute analytically the evolution of the system, the usual procedure of constructing the hierarchy of differential equations for the two spin moments is not useful. We have introduced a particle-hole description of the Ising model, in which the holes correspond to the domain walls (or "defects") between arrays of parallel spins. The energy of the system is directly related to the density of holes. Within this description, the present model has some similarities with the one-dimensional facilitated Ising model at zero temperature. Although the possible elementary dynamical events are different, both systems get eventually stuck in a metastable state characterized by all the holes being isolated, i. e., surrounded by two particles.

The particle-hole description allows us to solve analytically the time evolution of the system. A closed hierarchy of equations can be written for the probability distribution functions $D_{r}$ of finding $r+1$ consecutive holes in the system. The general solution of this hierarchy has been derived. In the long time limit, the system reaches a state in which $D_{r}=0$ for all $r \geq 1$, i. e., all the holes are isolated. Moreover, the asymptotic value of the density of holes is not unique, but it depends on the initial configuration.

Another similarity between the present model and the 1SFM shows up in the context of granular media. When both models are applied to study tapping processes, the steady state reached by the system is consistent with Edward's thermodynamic theory [32,33]. This has been shown numerically in Lefevre and Dean's model [28], and analytically for the 1SFM [30]. In the latter, an effective dynamics for the tapping process has been derived. This allows to find explicitly the steady distribution function of the system, as well as the relationship between the vibration intensity and Edwards' compactivity. Therefore, it seems worth trying to derive the corresponding effective dynamics for the tapping process in the model considered in this paper 34.

\section{ACKNOWLEDGMENTS}

We acknowledge partial support from the Dirección General de Investigación Científica y Técnica (Spain) through Grant No. PB98-1124.

[1] R. J. Glauber, J. Math. Phys. 4, 294 (1963).

[2] J. E. Anderson, J. Chem. Phys. 52, 2821 (1970).

[3] J. L. Skinner, J. Chem. Phys. 79, 1955 (1983).

[4] J. Budimir and J. L. Skinner, J. Chem. Phys. 82, 5232 (1985).

[5] H. Spohn, Commun. Math. Phys. 125, 3 (1989).

[6] J. J. Brey and A. Prados, Physica A 197, 569 (1993).

[7] J. J. Brey and A. Prados, Phys. Rev. E 53, 458 (1996).

[8] W. Kob and R. Schilling, J. Phys. A: Math. Gen. 23, 4673 (1990).

[9] J. J. Brey and A. Prados, Phys. Rev. B 49, 984 (1994).

[10] A. J. Bray, J. Phys. A: Math. Gen. 23, L67 (1990).

[11] A. Prados, J. J. Brey and B. Sánchez-Rey, Europhys. Lett. 40, 13 (1997).

[12] E. Lippiello and M. Zannetti, Phys. Rev. E 61, 3369 (2000).

[13] C. Godrèche and J. M. Luck, J. Phys. A: Math. Gen. 33, 1151 (2000).

[14] J. J. Brey and A. Prados, Phys. Lett. A 216, 240 (1996).

[15] W. Siewert and L. Schimansky-Geier, Phys. Rev. E 58, 2843 (1998).

[16] G. H. Fredrickson and H. C. Andersen, Phys. Rev. Lett. 53, 1244 (1984); J. Chem. Phys. 83, 5822 (1985).

[17] G. H. Fredrickson and S. A. Brawer, J. Chem. Phys. 84, 3351 (1986).

[18] J. Jäckle and S. Eisinger, Z. Phys. B 84, 115 (1991).

[19] S. Eisinger and J. Jäckle, J. Stat. Phys. 73, 643 (1993).

[20] J. Reiter and J. Jäckle, Physica A 215, 311 (1995). 
[21] E. Follana and F. Ritort, Phys. Rev. B 54, 930 (1996).

[22] F. Mauch and J. Jäckle, Physica A 262, 98 (1999).

[23] J. J. Brey, A. Prados and B. Sánchez-Rey, Phys. Rev. E 60, 5685 (1999).

[24] P. Sollich and M. R. Evans, Phys. Rev. Lett. 83, 3238 (1999).

[25] J. B. Knight, C. G. Frandrich, C. N. Lau, H. M. Jaeger and S. R. Nagel, Phys. Rev. E 51, 3957 (1995).

[26] E. R. Nowak, J. B. Knight, E. Ben-Naim, H. M. Jaeger and S. R. Nagel, Phys. Rev. E 57, 1971 (1998).

[27] N. G. van Kampen, Stochastic Processes in Physics and Chemistry (North-Holland, Amsterdam, 1981).

[28] A. Lefevre and D. S. Dean, J. Phys. A: Math. Gen. 34 L213 (2001).

[29] D. S. Dean and A. Lefevre, preprint cond-mat/0101166.

[30] J. J. Brey, A. Prados and B. Sánchez-Rey, Physica A 275, 310 (2000).

[31] D. S. Dean and A. Lefevre, preprint cond-mat/0106220.

[32] S. F. Edwards and R. B. S. Oakeshott, Physica A 157, 1080 (1989).

[33] A. Mehta and S. F. Edwards, Physica A 157, 1091 (1989).

[34] A. Prados and J. J. Brey (unpublished). 